\section{El perfil de salud-enfermedad bucal y las necesidades de tratamiento de los adultos mayores de Lima urbana 2012}

The profile of health - oral disease and treatment of urban Lima 2012 seniors needs

\section{Resumen}

El objetivo principal de este estudio fue determinar el perfil de salud- enfermedad bucal de las personas adultos mayores (AM) de Lima urbana, tomando en cuenta la prevalencia de caries dental, enfermedad periodontal, lesiones mucosa bucal y necesidades de tratamiento. Se realizó un estudio de tipo descriptivo-trasversal en 120 personas adultas mayores de 60 ańos, que viven en Lima cercado y distritos. La recolección de datos se realizó a través del método de observación clínica y entrevista haciendo uso de los índices y procedimientos de la OMS. Los resultados obtenidos fueron: edad promedio 71 años, con prevalencia del sexo femenino (70\%) y educación secundaria, presencia de hipertensión arterial $33 \%$ y diabetes miellitus $10.8 \%$, trastornos de la mucosa en un $10.8 \%$, siendo del tipo ulceración localizadas en el paladar y rebordes alveolares. Presencia de cálculos y sangrado periodontal, en el $52 \%$ de la población. El 23.3\% necesita profilaxis y solo un $11 \%$ raspaje radicular. De los dientes presentes en boca el $23 \%$ han perdido fijación en $4-5 \mathrm{~mm}$. Solo $5.82 \%$ de los dientes se encuentran sanos, siendo el promedio de caries 4.43 , dientes perdidos 14,77 del 53\%. El CPO tuvo una media de 19.71. Ds. 6.74. El 31.7\% usan prótesis total removible superior y prótesis fija en un 19,2\%. En el maxilar inferior un $20 \%$ utilizan PPR y el 13,3\% usan prótesis total y fija 9,27\%. Encontrándose una alta necesidad de prótesis removible en maxilar superior e inferior. Se logró determinar que la salud bucal de las personas adultas mayores es mala por efecto de la caries y enfermedad periodontal y con gran necesidad de higiene, operatoria y prótesis parcial removible, removibles totales y fijas.

Palabras clave: Adulto mayor, mucosa bucal, caries dental, índice CPO, prótesis dental, higiene bucal.

\begin{abstract}
The main objective of this study was to determine the profile of oral health and disease of the elderly adults of urban Lima, taking into account the prevalence of dental caries, periodontal disease, oral mucosa injuries and treatment needs. Descriptive and transversal study was using over 120 elderly adults (over 60 years old), living in Lima center and district. Data collection was performed by the method of clinical observation and interview using rates and procedures of the WHO. The results obtained were: average age 71 years, high prevalence of the female gender $(70 \%)$ and secondary education, presence of hypertension with $33 \%$ and diabetes mellitus with $10.8 \%$, the mucosal disorders in $10.8 \%$, with ulceration type localized on the palate and alveolar ridges. Also was observed presence of calculi and periodontal bleeding in $52 \%$ of the population. The study subjects requiring prophylaxis in $23.3 \%$ and needed just a case of root planing in $11 \%$. Teeth present in the mouth, the $23 \%$ have lost fixation at $4-5 \mathrm{~mm}$. Only $5.82 \%$ of the teeth are healthy, with an average of 4.43 cavities, missing teeth 14.77 with $53 \%$. The DMF had a mean of 19.71 with a Ds . 6.74. The $31.7 \%$ use removable full dentures and fixed prostheses by $19.2 \%$. In the lower jaw using PPR with $20 \%$ and $13.3 \%$ use full dentures and fixed prostheses with $9.27 \%$. Finding a high need for the use of removable dentures in upper and lower jaw. It was determined that the oral health of older people is poor due to the poor hygiene, introduction of caries and periodontal disease and in dire need of restoration of cavities, use of removable, removable total and fixed partial denture.
\end{abstract}

Keywords: Elderly, mouth mucosa, dental caries, DMF Index, dental prosthesis, oral hygiene.
Artículo Original
Carlos Campodónico Reátegui', Sylvia Chein Villacampa', Lourdes Benavente Lipa', Raúl Vidal Goñi', Juana Rosa Delgadillo Ávila², María Angélica Álvarez Paúcar ${ }^{3}$, Teresa Evaristo Chiyong' ${ }^{1}$, Carla Campodónico Morales ${ }^{5}$, Lourdes Lara Téllez ${ }^{4}$, Ana María Díaz Soriano ${ }^{1}$

1 Docente del Departamento Académico de Estomatología Preventiva y Social de la Facultad de Odontología de la UNMSM

2 Docente del Departamento Académico de Ciencias Básicas de la Facultad de Odontología de la UNMSM

3 Docente del Departamento Académico de Estomatología Pediátrica de la Facultad de Odontología de la UNMSM

4 Docente del Departamento Académico de Estomatología Rehabilitadora de la Facultad de Odontología de la UNMSM

5 Cirujano Dentista. Estudios Segunda Especialización Unidad de Postgrado de la Facultad de Odontología de la UNMSM

Correspondencia:

Mg. Carlos Campodónico Reátegui

Facultad de Odontología, Universidad Nacional Mayor de San Marcos.

Correo electrónico: ccampo_04umber@hotmail. com

Fecha de recepción: 14-08-2013

Fecha de aceptación: 27-09-2013

\section{Introducción}

El envejecimiento del ser humano es un proceso natural, universal, progresivo, irreversible y continuo durante todo el ciclo de vida. La calidad de vida y la funcionalidad durante la vejez están directamente relacionadas con las bases genéticas de la persona, los estilos de vida, la calidad del medio ambiente, el acceso y calidad de los servicios de salud y a las enfermedades sufridas. Según ENAHO (2001), las enfermedades crónico degenerativas hipertensivas, artrosis y diabetes mellitus, alteraciones en la funcionalidad o dependencia física, sitúan a los AM en una posición de vulnerabilidad; con débil soporte familiar y social, lo sitúan como desprotegido. ${ }^{1,2}$ En relación con la salud estomatológica de los ancianos en México, reportes indican que existen problemas severos de edentulismo, caries coronal y caries radicular. La poca utilización de servicios 
y la indiferencia en el cuidado de las prótesis removibles por lo ancianos provoca que éstas se encuentren en malas condiciones, y con ello lesiones en los tejidos bucales ${ }^{3}$ Irigoyen y col. (1999), estudio en personas AM en México, en las delegaciones Alvaro Obregón y Tlahuac, mostraron un CPOD de 16.3. El componente más elevado correspondió a los dientes perdidos 9.0 seguido por los cariados 4.6 y por último los obturados con $2.7^{4}$.

En el Japón, un 37,6 \% de las personas de la tercera edad postrados necesitan una prótesis total o parcial. ${ }^{5}$ En Turquía el 32,6\% de los pacientes dentados presentaron 2,2 piezas con caries radiculares y el 20,7 \% tenía al menos una lesión cariosa en corona no tratada. ${ }^{6}$ En Chile $48.3 \%$ era desdentado total y $51.7 \%$ parcial, esto se distancia un poco de lo obtenido en adultos mayores de 60 años de nivel socioeconómico bajo en el norte de Santiago en los cuales el $25,9 \%$ era desdentado total. ${ }^{8}$ Las personas con un edad mayor de 81,8 ańos, presentaron una mayor pérdida de piezas dentarias. Todos necesitaban prótesis dental, ya sea porque no poseían o las que tenían se encontraban desajustadas. El 48,3 \% no contaba con prótesis a diferencia de lo mostrado por Misrachi en adultos mayores de 60 años de nivel socioeconómico bajo en el norte de Santiago donde el 18,6 \% no contaba con prótesis. ${ }^{7,8}$ Se estudió a 540 ancianos, los principales problemas en las actividades habituales fueron comer (14.4\%), hablar (8.7\%), incapacidad de no irritarse (5.4\%), lavarse los dientes $(5 \%)$ e imposibilidad de disfrutar el contacto con la gente $(4.4 \%)$. Existe una correlación del OIDP positiva entre el número de dientes perdidos y el índice CPO-D $(\mathrm{p}<0.05)$ y negativa entre el número de dientes funcionales y los obturados $(\mathrm{p}<0.05) .{ }^{9,10}$

En un estudio en 234 personas adultas mayores de ambos sexos, se encontró que el $53 \%$ habían perdido la totalidad de sus dientes y el 38,4\% con pérdida dental parcial que requiere de rehabilitación protésica para satisfacer la capacidad masticatoria. De las personas que conservan al menos un diente en boca, la caries activa es prevalente en el 77,7 $\%$ y las periodontopatías en el 52,9\% de esta población y el 83,5\% tiene un deficiente control de la placa dental. El 63,9 \% de las prótesis superiores y el 74,2 \% de las prótesis inferiores necesitan ser reacondicionadas o renovadas por prótesis bien concebidas. ${ }^{11}$ Los malos estilos de vida asociados a la deficiente nutrición y a las malas prác-

ticas de autocuidado, son otro factor a ser considerado en la etiopatología de la morbilidad dental de las personas adultas mayores. También hay que tomar en cuenta que la mala higiene de la boca y de las prótesis dentales no solamente es un factor de riesgo de las enfermedades odontológicas, sino de las infecciones neumónicas. ${ }^{12}$

En el Perú, Lima la capital, la situación de salud - enfermedad de los adultos mayores, no difiere con la de otros países latinos donde, la mala higiene, la dieta está íntimamente relacionada con la pérdida dentaria, caries múltiple, necesidad de tratamientos y de prótesis PPR, prótesis total removible.

Esta investigación es necesaria, porque el conocimiento del perfil de salud del adulto mayor va permitir, establecer programas preventivos desde etapas de vida temprana para preveer la salud bucal; así como instalar programas para la rehabilitación de la salud bucal de los adultos mayores.

\section{Material y métodos}

El presente trabajo fue un estudio cuantitativo, epidemiológico, de corte trasversal, descriptivo observacional, que permitió analizar las características de las enfermedades bucales y necesidad de tratamiento de la población de adultos mayores de la ciudad de Lima. La población de estudio estuvo conformada por personas adultos mayores de 60 años de edad de la ciudad de Lima; dicho estudio se realizó en una población conformada por centros de protección del adulto mayor como: asilos, centros de protección de los hospitales de ESSALUD, MINSA, Clubs de tercera edad de Lima. La muestra fue seleccionada en forma aleatoria siendo de 120 personas. Se trabajó con un nivel de confianza de un $95 \%$ y de preci-

Tabla 1. Estado periodontal de los adultos mayores

\begin{tabular}{ccc}
\hline Estado Periodontal & Frecuencia & Porcentaje \\
\hline Sano & 14 & 11.7 \\
Sangrado & 16 & 13.3 \\
Calculo & 26 & 21.7 \\
Bolsa 4-5 mm & 10 & 8.3 \\
Bolsa más de 6 & 5 & 4.2 \\
No registrado & 49 & 40.8 \\
Total & 120 & 100 \\
\hline
\end{tabular}

Se encontró una media de 5,82 y una Ds. 5.71 con un valor mínimo de 00 y máximo 23 con una moda de 00 a 2 piezas de dientes sanos, mientras que los dientes cariados presentaron una sión del $5 \%$. Uno de los criterios de exclusión fueron personas en estado de gravedad con enfermedades terminales. Para la recolección de datos participaron docentes de la Facultad, alumnos de pre y post grado, previamente calibrados. Se empleó los índices CPOD e IP, también los instrumentos físicos determinados por la OMS (sondas periodontales, exploradores, curetas, espejos). Se empleó la técnica de entrevista y la observación clínica, en un ambiente de la institución previamente acondicionado para tal fin. Los datos se registraron en una ficha preparada para esta investigación.

\section{Resultados}

La edad de los adultos mayores del estudio, estaba comprendido entre 60 y 91 años de edad con una media de 71,20 años Ds 7,86 y una moda de 60 años siendo el grupo de 60 a 65 años el más frecuente $36 / 120$ con un predominio del sexo femenino 70 \%. El 56,7 \% presentan un grado de instrucción secundaria. El 51.7 \% proceden de Lima y el 25.85 de la Sierra. El $33.3 \%$ de la población de adultos mayores presentan hipertensión arterial y un $10.8 \%$ diabetes mellitus. Dentro de los trastornos de la mucosa lo más común son la lesiones ulcerativas en un $10.8 \%$, pero un $67.5 \%$ no padecía ninguna lesión en la cavidad bucal. La localización de las lesiones orales la más frecuente fue el paladar en $10 \%$ y bordes alveolares. Con respecto al estado periodontal lo más frecuente fue cálculos en un 21,7 $\%$ y sangrado en un $13.3 \%$. El 23,3 $\%$ requiere educación de la salud, profilaxis y un $11 \%$ además necesita raspaje. La pérdida de fijación de los dientes presentes en boca fue de o- $3 \mathrm{~mm}$ en el $31 \%$ de los AM, seguido de 4-5 mm en un $23 \%$.

media 4,43 y Ds. $2 . .98$ con valores 1 a 20. Los dientes perdidos en promedio fueron 14,77 y Ds. 8,54 y moda 5-8 piezas. El promedio de los dientes obturados fue media 5. 5 Ds. 4,5. 
Tabla 2. CPO-D en los adultos mayores de Lima 2012

\begin{tabular}{ccc}
\hline CPO & Frecuencia & Porcentaje \\
\hline $2-7$ & 7 & 5,8 \\
$8-10$ & 6 & 5,0 \\
$11-13$ & 12 & 10,0 \\
$14-16$ & 17 & 14,2 \\
$17-19$ & 9 & 7,5 \\
$20-22$ & 20 & 16,7 \\
$23-25$ & 19 & 15,8 \\
$26-28$ & 30 & 25,0 \\
Total & 120 & 100,0 \\
\hline
\end{tabular}

El CPO-D promedio fue 19.71 y Ds. 6.74 con una moda de 26 a 29 dientes afectados por caries. El $39 \%$ de los pacientes presentaron de 1-14 coronas, la mayor frecuencia fue 1-2 coronas con un 43,6 \%. Solo se encontró que 7 personas habían perdido dientes por razones diferentes a caries dental. Se encontró que 14 adultos mayores tenían dientes traumatizados entre 1 y 11 piezas.

44 personas (56.8 \%) necesitan obturar entre 1 y 2 piezas. Con una media de 2,75 Ds. 1.93. 47 personas necesitan obturar dos o más superficies dentarias. 17 necesita obturar un diente, $73 \%$ necesitan obturar hasta 3 dientes.

De los 120 adultos mayores solo 17 necesitan coronas dentarias, de los cuales el 70,6\% requiere entre 1 a 2 coronas.

La necesidad de tratamiento pulpar sólo la presentaron 8 personas de 120 adultos mayores de la muestra. La necesidad de extracciones dentarias fue $10 \%$, de las cuales el $50 \%$ necesita extraer una pieza.

Tabla 3. Situación de la prótesis en el maxilar superior e inferior en adultos mayores

\begin{tabular}{ccccccc}
\hline \multirow{2}{*}{ Situación de Prótesis } & \multicolumn{2}{c}{ Prótesis Superior } & \multicolumn{2}{c}{ Prótesis Inferior } & \multicolumn{2}{c}{ Total } \\
& $\mathrm{f}$ & $\%$ & $\mathrm{~F}$ & $\%$ & $\mathrm{f}$ & $\%$ \\
\hline Ninguna Prótesis & 32 & 26,7 & 57 & 47,5 & 89 & 37,0 \\
Prótesis Fija (PF) & 23 & 19,2 & 11 & 9,2 & 34 & 14,0 \\
Prótesis Parcial Removible (PPR) & 14 & 11,7 & 24 & 20,0 & 38 & 15,0 \\
PF+PPR & 13 & 10,8 & 12 & 10,0 & 25 & 10,4 \\
Prótesis Completa R & 38 & 31,0 & 16 & 13,3 & 54 & 22,5 \\
Total & 120 & 100,0 & 120 & 100,0 & 240 & 100,0 \\
\hline
\end{tabular}

De las personas estudiadas un 26.7 \% no son portadores de algún tipo de prótesis superior, mientras un 31.7 \% son portadores de prótesis completa removible superior seguido de prótesis fija en un 19,2\%. Sobre la situación del uso de prótesis inferior, hay un $47,5 \%$ de la población que no usan ningún tipo de prótesis, hay $20 \%$ que utilizan PPR y el 13,3 \% usan prótesis completa removible.

Tabla 4. Necesidad de prótesis en el maxilar superior e inferior en adultos mayores Lima 2012

\begin{tabular}{ccccccc}
\hline Necesidad de Prótesis & \multicolumn{2}{c}{ Prótesis Superior } & \multicolumn{2}{c}{ Prótesis Inferior } & \multicolumn{2}{c}{ Total } \\
\hline Ninguna & $\mathrm{f}$ & $\%$ & $\mathrm{~F}$ & $\%$ & $\mathrm{f}$ & $\%$ \\
Coronas & 44 & 36,7 & 34 & 28,3 & 78 & 32,5 \\
Prótesis Fija & 00 & 0,0 & 4 & 3,3 & 4 & 1,6 \\
PPR & 14 & 11,7 & 10 & 8,3 & 24 & 10,0 \\
PF+PPR & 30 & 25,0 & 51 & 42,5 & 81 & 33,7 \\
Prótesis Completa R & 2 & 1,7 & 1 & 0,8 & 3 & 1,2 \\
No registrable & 28 & 23,3 & 20 & 16,7 & 48 & 20 \\
Total & 2 & 1,7 & 00 & 0,0 & 2 & 0,8 \\
\hline
\end{tabular}


Necesidad de prótesis superior: PPR requiere un $25 \%$, prótesis total removible un $23.3 \%$, el 36,7 \% no necesita ningún tipo de prótesis Sobre necesidades de prótesis inferior en adultos mayores, un $42.5 \%$ requiere PPR;-Prótesis Total necesita un $16.7 \%$, el $28,3 \%$ no demanda ninguna.

Tabla 5. Necesidad de prótesis total superior e inferior en adultos mayores

\begin{tabular}{ccc}
\hline Necesidad Total de Prótesis & Frecuencia & Porcentaje \\
\hline Si & 13 & 10,8 \\
No & 107 & 89,2 \\
Total & 120 & 100,0 \\
\hline
\end{tabular}

La necesidad de ambas prótesis total superior e inferior solo es del $10.8 \%$. La higiene bucal en los adultos mayores, solo se pudo medir al $49 \%$ de las personas, el resto tenían los dientes suficientes para aplicar el IHO simplificado, donde lo más prevalente es una mala higiene en un $31 \%$ seguido de una higiene regular en un $28 \%$.

\section{Discusión}

En nuestro estudio, la muestra estuvo conformado por personas comprendidas entre 60 y 91 años de edad con una media de 71,20 años Ds 7,86, con una prevalencia del sexo femenino 70 $\%$. Las enfermedades que padecen los adultos mayores (AM) son la hipertensión arterial con un $33.3 \%$ y un 10.8 $\%$ diabetes mellitus. Según Snow y col. ${ }^{11}$ determinaron que la diabetes en los AM se presenta con mayor frecuencia que en otros grupos etarios, el Ministerio de Salud del Perú ${ }^{1}$ determinó que los AM sufren de diabetes mellitus e hipertensión arterial, estos resultados son muy parecidos a los resultados obtenidos de Lima. Dentro de los trastornos de la mucosa lo más común encontrado fueron las lesiones ulcerativas en un $10.8 \%$, donde el $67.5 \%$ se encontraban mayormente en el paladar, no encontramos estudios previos sobre el trastorno de la mucosa de los adultos mayores.

Sobre el estado periodontal, lo más frecuente que se encontró fue cálculos dentales en un $21,7 \%$ y sangrado gingival en un $13.3 \%$. Otras investigaciones acerca del estado periodontal, las relacionan en forma general mas no son específicas (según la OMS). Sobre el tratamiento periodontal de adultos mayores un 52,5\% no necesitan de tratamiento por múltiples motivos, generalmente la perdida de la dentadura, 23,3\% necesita educación de la salud, profilaxis y un $11 \%$ además necesita raspaje. No existen trabajos de investigación que permita hacer las comparaciones.

En cuanto a los dientes sanos en los AM, se encontró una media de 5,82 y una Ds. 5.71, muy baja, la dentadura de los adultos mayores, llega muy afectada a los 60 años, principalmente por caries, segundo por perdida por enfermedad periodontal, encontrando personas con 00 dientes en boca y con un máximo de 23 sanas en muy escaso número, lo más común son personas con 00 a 2 piezas dentarias. En otros contextos de América Latina, como es el caso de Chile, la frecuencia de pérdida dentaria en personas que asisten a clubes de ancianos en Valparaíso fue de $33 \%$ y se encontró que aquellos que aún conservaban dientes tenían un promedio de seis dientes (16) como el caso de Cuba, donde el 9,4\% de la población mayor de 65 años, conservaba más de 20 dientes. $^{16}$

El análisis de la caries dental encontrada en adultos mayores, en promedio fue de 4.43 con una variación de 2.98 , lo más frecuente fue una caries, los valores encontrados de 1 y 20 piezas afectadas. Se determinó que esta enfermedad tiene una prevalencia muy alta, esto también sucede en otras investigaciones de otros países como México 5 donde el promedio de caries fue de 4,6 piezas afectadas muy parecido a lo que sucede en LimaPerú ${ }^{1}$. En el estudio del cantón de Barva de Heredia, solamente el $4 \%$ mostró 20 o más piezas dentales en boca. La frecuencia de caries dental, medida por el Índice COPD (número de dientes cariados, obturados y perdidos) reportados en cinco países latinoamericanos, todos ellos realizados con personas mayores de 60 años, reportaron los siguientes datos ${ }^{14}$

Sobre los dientes perdidos por caries, encontramos que el promedio fue de de la perdida más frecuente fue de 28 14,77 y una variabilidad de 8,54 don- piezas dentarias, encontrando personas con una hasta 28 piezas (10.8\%) dientes perdidos. En estudios realizados en México el promedio fue de 9 piezas perdidas (5) en Chile en la ciudad de Santiago se encontraron adultos mayores que han perdido 28 piezas en un $25.9 \%$ con un promedio de vida de 81 ańos 7 . Lo semejante de estos estudios con el nuestro, es que la pérdida dentaria es elevada, siendo los promedios diferentes, la cifra mucho mayor es, en Lima.

El efecto de la caries dental en los adultos mayores de Lima se determinó con el CPO-D, donde la media fue 19.7 piezas, la más alta frecuencia fue de 26 a 29 piezas afectadas. En otras investigaciones como en México el CPO tuvo una media fue de 16.3 piezas $^{4}$. La frecuencia de caries dental, medida por el Índice COPD (número de dientes cariados, obturados y perdidos) reportados en cinco países latinoamericanos, todos ellos realizados con personas mayores de 60 ańos, reportaron los siguientes datos: Brasil 27,2, Costa Rica 26,1, Cuba 28,0, Chile 24,7 y Venezuela 19,1. El componente pieza dental perdida, fue el más frecuente del Índice COP-D. Haciendo una comparación con países latinos, en Lima encontramos que es más bajo el CPO, de repente porque nuestro estudio tomó en consideración personas con un nivel de educación media, y no se encontraban en extrema pobreza, la mayoría habían sido trabajadores de oficina.

De las personas estudiadas un $26.7 \%$ no son portadores de algún tipo de prótesis superior, un $31.7 \%$ tiene prótesis removible superior seguido de prótesis fija en un 19,2 \%, en relación a las prótesis inferior un $20 \%$ usan PPR y un $13,3 \%$ usan prótesis total removible, un $47.5 \%$ no usan algún tipo de prótesis; acerca de la necesidad de prótesis superior un $25 \%$ lo requieren, un 23,3 $\%$ demanda prótesis total removible. En relación a la necesidad de prótesis inferior un 42,5\% necesita de prótesis PPR y un 16,7 \% prótesis completa removible. En cuanto a la necesidad de prótesis completa removible superior e inferior es de 10,8\%.

En relación a la higiene bucal de estos adultos mayores; el $42,5 \%$ tenía falta de dientes, no se pudo medir; al 57,5\% se pudo medir la placa, en un $25 \%$ se encontraron con mala higiene y $23 \%$ regular, parecidos a estudios realizados en Latinoamérica ${ }^{16,17}$ 


\section{Conclusiones}

Los adultos mayores estudiados fueron predominantemente del género femenino, que cursaron educación secundaria a superior. Ellos padecen de diabetes e hipertensión arterial en un alto porcentaje.

Respecto a las enfermedades bucales que padecen los AM, se registraron mayormente lesiones en mucosa (ulceras) en la zona del paladar. Sobre la enfermedad periodontal, este grupo registró una higiene bucal mala, fue más común hallar gingivitis con sarro dental, además se registró pérdida de soporte dental moderado. El estado dental es muy malo, por una elevada pérdida de piezas dentales por causa de la caries, asimismo se observó en su mayoría caries dental activa.

Los AM registraron ser portadores de prótesis parcial removible superior e inferior, seguido de portadores de prótesis fija, además se observó a un bajo porcentaje que usaron prótesis total removible. Mientras que, las necesidades de tratamiento en este grupo, son principalmente el uso de las prótesis parciales removible superior e inferior, prótesis fijas y un bajo porcentaje necesita prótesis total removible superior e inferior.

El equipo de investigación, recomienda realizar un estudio sobre las limitaciones psicológicas (depresión, otros) y la higiene bucal de los adultos mayores, (abandono de la salud bucal) relacionado a la situación socio-económica y las enfermedades bucales. Asimismo, recomendamos desarrollar un programa de salud bucal para los adultos mayores.

\section{Referencias Bibliográficas}

1. Ministerio de salud del Perú. Dirección general de salud de las personas. Lineamientos para la atención Integral de Salud de las Personas Adultas mayores. Perú 2005
2. Instituto Nacional de Estadística Geografía e Informática México. Resultados preliminares Censo de 2005.

3. OPS. Guía Clínica para Atención Primaria a las Personas Adultas Mayores. Tercera Edición. Enero. 2003

4. Irigoyen ME, Velásquez C. Zepeda MA, Mejía A. Caries dental y enfermedad parodontal un grupo de personas de 60 o más años de edad de la CD. de México. Rev. Asoc. Dent. Mex. 1999;56(2):64-69.

5. Morishita M., et al. Oral health care status of homebound elderly in Japan. Journal of Oral Rehabilitation, 2001;28(8):717-20.

6. Unlüer $S$, Gökalp S, Doğan BG. Oral health status of the elderly in a residential home in Turkey.Gerodontology, 2007;24(1):22-9.

7. Misrachi C, Cabargas J. Salud Oral en el Adulto Mayor. Santiago, Chile. Universidad de Chile; 2005. 17-26 p.

8. Araya C, Oliva P, Canete E, Zapata H. Ciencia- Ahora 2009; 23, año 12 , enero a junio.

9. Sánchez-García $S$, et al. Estado de la dentición y sus efectos en la capacidad de los ancianos para desempeñar sus actividades habituales. Salud pública Méx [Internet]. 2007 [Citado 11 de julio de 2012];49(3):173-181. Disponible en: http://www.scielo.org.mx/pdf/ spm/v49n3/02.pdf

10. Brenes W, Hoffmaister F. Situaciones de salud bucal en personas adultas mayores: Problemas y desafíos. Rev. costarric. cienc. méd [Internet]. 2004 [Citado 11 de julio de 2012];25 (3-4): 27-41. Disponible en: http://www.scielo. sa.cr/scielo.php?pid=S025329482 004000200003\&script=sci_arttext
11. Snow CM, Martín CC, Shaw JM: Physical activity and risk for osteoporosis. In: Marcus R, Felman D, Kelsey J (eds): Osteoporosis. San Diego, Academic Press; 1996. 511$525 \mathrm{p}$.

12. Yoneyama T, Yacida M, Obrui T, et al. Oral care deduces pneumonia in older patients in nursing homes. Amer Geriat Soc. 2002; 50(3):431.

13. Brenes W, Murillo G. Estado de salud oral y utilización de los servicios odontológicos. Estudio de la tercera edad Monografía 90-01 / Serie: Salud del Adulto Costa Rica. San José 1990. 97-111 p.

14. Murillo O. Diferencias en las condiciones bucodentales en hombres y mujeres del canton de Barva. Anales en Gerontología, Revista de la Maestría Interdisciplinaria en Gerontología de la Universidad de Costa Rica 1999;1(1):69-77.

15. Badenier O, Mariño R. Magnitud de las enfermedades dentales en adultos mayores de 60 ańos. En: Vejez y envejecimiento. Vol.2. Santiago,Chile. Universidad de Chile; 1987. 34-7 p.

16. Ministerio de Salud. Estado del cumplimiento de las metas nacionales de salud bucal para el año 2000. Ministerio de Salud Pública. Dirección Nacional de Estomatología. Cuba 1989.

17. Marińo R. La salud bucodental de los ancianos: realidad, mitos y posibilidades. Bol Of Sanit Panam 1994; 116(5):419-26. 